\title{
The cytotoxic effects of lipidic formulated gold porphyrin nanoparticles for the treatment of neuroblastoma
}

This article was published in the following Dove Press journal:

Nanotechnology, Science and Applications

15 June 2010

Number of times this article has been viewed

\author{
Puiyan Lee' \\ Yifan Zhu' \\ Jessie J Yan ${ }^{2}$ \\ Raymond WY Sun ${ }^{2}$ \\ Wei Hao' \\ Xuelai Liu' \\ Chi-Ming Che ${ }^{2}$ \\ Kenneth KYWong' \\ 'Department of Surgery, Li Ka Shing \\ Faculty of Medicine, ${ }^{2}$ Department \\ of Chemistry and Open Laboratory of \\ Chemical Biology of the Institute \\ of Molecular Technology for Drug \\ Discovery and Synthesis, The \\ University of Hong Kong, Hong Kong
}

Correspondence: Kenneth KYWong Department of Surgery, The University of Hong Kong, Queen Mary Hospital, Pokfulam Road, Hong Kong SAR

Fax +852 28I73I55

Email kkywong@hku.hk
Objective: Nanotechnology has been identified as a promising platform in the improvement of the design and development of drug delivery systems. In the present study we investigated the potential of lipidic nanoparticles consisting of gold porphyrin for the treatment of neuroblastoma.

Materials and methods: To characterize the size of the lipidic gold porphyrin nanoparticles, we used transmission electron microscopy (TEM). The in vitro cytotoxic effect on neuroblastoma activity was examined using XTT cell proliferation assay, then $\mathrm{IC}_{50}$ values were calculated. In vivo safety and toxicity were studied using intraperitoneal injection of gold porphyrin nanoparticles into normal animals. Finally, tumor size measurement and animal survival were studied to investigate the therapeutic effect of lipidic gold porphyrin nanoparticles on neuroblastoma growth.

Results: We found that incorporation of gold porphyrin into lipidic nanoparticles resulted in a 16-fold increase in size. Subsequent in vitro and in vivo cytotoxicity studies further showed that the lipidic gold porphyrin nanoparticles could decrease systemic toxicity, as well as inhibiting tumor growth following administration into the neuroblastoma bearing mice.

Conclusion: The delivery of lipidic gold porphyrin nanoparticles by incorporation with lipidic formulation is feasible approach to treat neuroblastoma. We await further studies to evaluate tumor killing kinetics.

Keywords: nanoparticles, lipidic formulation, gold porphyrin, neuroblastoma

A delivery system that is capable of reducing toxicity improves the design in formulation of cancer therapeutics. This is especially true when treatment is required for long term administration, for example in neuroblastoma, which is a cancer of the sympathetic nervous system. It is the most common solid tumor in children, making up approximately $7 \%$ of all childhood tumors. ${ }^{1}$ Despite advances in medical technology, the overall tumor-related survival rate across all stages is still only around $50 \%$. Furthermore, because of minimal symptoms at the early stages, many neuroblastoma cases are diagnosed after the cancer has metastasized to other parts of the body. Thus, a common treatment option for neuroblastoma is high dosages of chemotherapy and/ or radiotherapy. ${ }^{2}$ However common chemotherapeutics for treating neuroblastoma, such as cisplatin, can cause significant systemic side effects. ${ }^{3}$

Neuroblastoma is known to have a disorganized vascular network. ${ }^{4}$ Despite the presence of leaky blood vessels supplying the tumor, clearance can be slow due to slow lymphatic drainage. ${ }^{5,6}$ Macromoleculer therapeutics, such as nanoparticles, therefore tend to accumulate in the tumor. The concept of enhanced permeability and retention (EPR) is the standard for the development and design of lipidic, micellar, or polymeric 
anticancer therapeutics. Results of preclinical studies have found that the nanoparticle based delivery system shows promise as a selective tumor targeting strategy by utilizing the abnormal anatomical structure in the tumor. ${ }^{7-9}$

The recently developed gold(III) porphyrin complex (gold-1a) forms a new generation in gold(III)-based cancer chemotherapeutics. ${ }^{10,11}$ Unlike the first generation anticancer gold compounds, which are unstable in physiological conditions, the tetradentate porphyrinato ligand of gold-1a stabilizes the gold(III) ion against reduction and demetalation. ${ }^{12}$ In vitro and preclinical studies have demonstrated its potency against a wide range of cancers such as nasopharyngeal carcinoma and hepatocellular carcinoma. ${ }^{13}$ It was recently reported that gold porphyrin is a potent inhibitor of thioredxin reductase that is responsible for regulating the overall intracellular reduction/ oxidation balance. ${ }^{14}$ It can be a more potent antitumor agent than currently available chemotherapeutic agent, cisplatin, with a maximum at 100 fold higher cytotoxicity. ${ }^{13}$ It has also been suggested that the possible apoptotic mechanism of cancerous cells might involve the caspase-signaling pathway. ${ }^{15}$ Gold(III) porphyrin also greatly affects the function of mitochondria and results in cell apoptosis. ${ }^{16}$

As has been demonstrated in most traditional chemotherapeutics, undesirable side effects in nontumoral tissue are often due to the high toxicity contained in these treatments. The present study was aimed at exploring the feasibility of a nanotechnology-based approach for the delivery of gold porphyrin. We designed lipidic gold porphyrin nanoparticles by incorporating the free gold porphyrin into a lipidic formulated carrier and evaluated the in vitro and in vivo toxicity. The lipidic based nanoparticle carriers were made of cetyl alcohol and the resulting nanoparticle was determined to be safe and biocompatible with the great blood compatibility based on the report by Mumper et al. ${ }^{17}$ To prolong the circulation duration in the in vivo system, we further incorporated poly(ethylene) glycol (PEG) into the nanoparticles as previously described. ${ }^{18}$ In order to evaluate the in vivo efficacy of lipidic gold porphyrin nanoparticles, we examined the antitumor activity in the $\mathrm{A} / \mathrm{J}$ neuroblastoma mouse model.

\section{Materials and methods Neuroblastoma cell lines}

Five human neuroblastoma cell lines LAN-1, LAN-2 (ICLC, Genova, Italy), SH-SY5Y, Be(2)-C, CHP-212 (ATCC, Manassas, VA) were cultured in MEM and a mouse neuroblastoma cell line N2A (ATCC, Manassas, VA) was cultured in DMEM according to the manufacture protocol.

\section{Synthesis of lipidic gold porphyrin nanoparticles}

Gold(III) porphyrin complex (gold-1a) was kindly provided by the Department of Chemistry, HKU. The lipidic gold porphyrin nanoparticles were prepared as previously described using oil in water $(\mathrm{o} / \mathrm{w})$ microemulsion. ${ }^{19}$ Briefly, $1 \mathrm{~mL}$ of lipidic gold porphyrin nanoparticles was synthesized by completely dissolving the gold porphyrin in the oil phase of cetyl alcohol at $60^{\circ} \mathrm{C}$. Surfactant Brij 78 was added to the oil phase and stirred for 5 minutes. To form the microemulsion template, $1 \mathrm{~mL}$ of deionized water was added and stirred well for 20 minutes. The microemulsion template was cooled at room temperature for 10 minutes to form lipidic gold porphyrin nanoparticles. The size of lipidic gold porphyrin nanoparticles was characterized using transmission electron microscopy (TEM). Briefly, the specimen on the supporting copper grid was prepared by dipping into free gold porphyrin nanoparticles in ethanol or lipidic gold porphyrin nanoparticles in water solution. The samples were dried on a copper wire and placed in a vacuum chamber for imaging.

\section{In vitro cytotoxicity assay}

The six neuroblastoma cell lines LAN-1, LAN-2, SH-SY5Y, $\mathrm{Be}(2)-\mathrm{C}, \mathrm{CHP}-212$, and N2A were seeded in a 96 well plate and incubated with free gold porphyrin nanoparticles or lipidic gold porphyrin nanoparticles for 6 hours. Following incubation, the XTT (Sodium 3,3'-(-[(Phenlamino)carbonyl]3,4-tetrazolium)-bis(4-methoxyl-6-nitro)benzene sulfonic acid hydrate) colorimetric assay was performed according to the manufacturer's protocol (Roche Diagnostics, Indianapolis, $\mathrm{IN}$ ) and $\mathrm{IC}_{50}$ values, measured as the concentration of drug required to inhibit $50 \%$ of the cell growth, were calculated by constructing a dose dependent curve. ${ }^{13}$ XTT is a quantitative colorimetric assay based on the development of an orange formazan dye by viable cells from the cleavage of the yellow XTT tetrazolium salts. Briefly, $10^{4}$ of individual cells were seeded in each well of a 96 well plate and cultured at $37^{\circ} \mathrm{C}$ in a $5 \% \mathrm{CO}_{2}$ incubator. A negative control using the cell culture medium only was taken as the blank. XTT assay was performed using the ELISA plate reader with absorbance at $480 \mathrm{~nm}$ and the reference absorbance at $650 \mathrm{~nm}$ as the filter according to the protocol provided from the commercial kit (Roche Diagnostic, Indianapolis, IN).

\section{In vivo efficacy of anticancer activity in the neuroblastoma model}

Six to eight week old male inbred $\mathrm{A} / \mathrm{J}$ albino mouse strain widely used in immunology and cancer study, weighing 
$20 \pm 2 \mathrm{~g}$ were obtained from and housed in the Laboratory Animal Unit at The University of Hong Kong. Experimentation protocol was approved by the Committee on the Use of Live Animals for Teaching and Research, University of Hong Kong (CULATR case no. 1901-09). A $200 \mu 1$ mouse neuroblastoma cell line $(\mathrm{N} 2 \mathrm{~A})$ with the density of $1.5 \times 10^{6} / \mathrm{ml}$ resuspended in PBS was subcutaneously inoculated on the right flank of $\mathrm{A} / \mathrm{J}$ mouse. At 11 days after inoculation, mice were either untreated or treated with intraperitoneal injection of $0.08 \mathrm{mg}$ or $0.1 \mathrm{mg}$ (approximately 4 or $5 \mathrm{mg} / \mathrm{kg}$ of mouse) of lipidic gold porphyrin nanoparticles every 7 days $(n=6)$. Measurement of tumor volume was conducted every 3 days for a total of 21 days. For the survival rate study, the lipidic gold porphyrin nanoparticles at $0,0.04,0.06,0.08,0.1$, and $0.3 \mathrm{mg}$ (approximately $0,2,3,4,5,15 \mathrm{mg} / \mathrm{kg}$ of mouse) or free gold porphyrin nanoparticles at $0.1 \mathrm{mg}$ (approximately $5 \mathrm{mg} / \mathrm{kg}$ of mouse) was intraperitoneally injected into the mouse.

\section{Statistical analysis}

Data was analyzed using the parametric Student's $t$ test and $P<0.05$ was taken the level of statistical significance.

\section{Results}

\section{Size determination of gold porphyrin nanoparticles}

TEM was used to determine the average size of free gold porphyrin nanoparticles and lipidic gold porphyrin nanoparticles. The average diameter of free gold porphyrin nanoparticles was approximately $2-5 \mathrm{~nm}$ and of lipidic gold porphyrin nanoparticles was approximately $80 \pm 30 \mathrm{~nm}$. This indicates that incorporation of lipid coating resulted in a 16-fold increase in size (Figure 1).

\section{In vitro toxicity}

An XTT assay was conducted to evaluate the in vitro antitumor activity of free gold porphyrin nanoparticles and lipidic

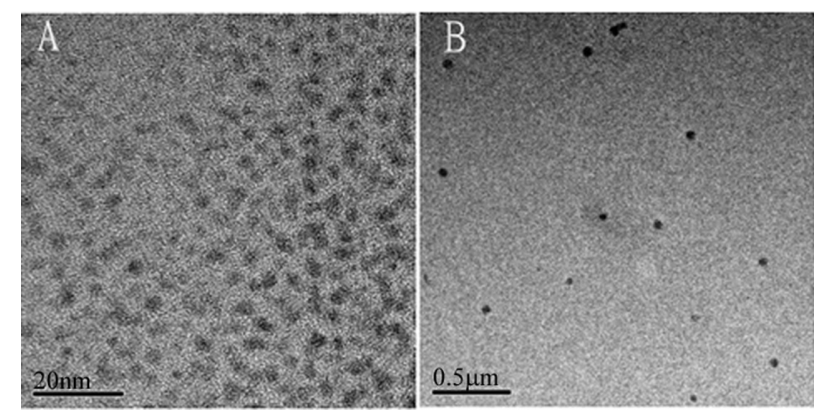

Figure I Size characterization by transmission electron microscopy (TEM) of A) free gold porphyrin, or B) lipidic gold porphyrin nanoparticles. The scale bar represents $20 \mathrm{~nm}$ or $0.5 \mu \mathrm{m}$. gold porphyrin nanoparticles. Both free gold porphyrin nanoparticles and lipidic gold porphyrin nanoparticles demonstrated positive cytotoxicity against all six neuroblastoma cell lines: N2A, CHP-212, Be(2)-C, SH-SY5Y, LAN-1, and LAN-2. The $\mathrm{IC}_{50}$ values showed that the cytotoxicity of both lipidic gold porphyrin nanoparticles and free gold porphyrin nanoparticles against the six neuroblastoma cell lines was dose dependent (data not shown). The $\mathrm{IC}_{50}$ value determined by the dose dependent curve of lipidic gold porphyrin nanoparticles was higher in N2A, CHP-212, Be(2)-C, SH-SY5Y, while it was lower in LAN-1 and LAN-2 when compared with free gold porphyrin nanoparticles (Table 1). This would suggest that various cell lines had different sensitivity to the lipidic gold porphyrin nanoparticles.

\section{In vivo cytotoxicity}

For the design of a nanoparticle with a long half-life in the systemic circulation in vivo, PEG was used to coat the nanoparticles. The 28 day survival study showed that $100 \%$ of the mice survived after receiving intraperitoneal injection of $0.02,0.04,0.06,0.08,0.1$, or $0.3 \mathrm{mg}$ of lipidic gold porphyrin nanoparticles (Figure 2). In contrast, the survival rate dropped to $50 \%$ at day 6 after $0.1 \mathrm{mg}$ of free gold porphyrin nanoparticles was administered. This indicated the lipidic nanoparticle formulation reduced the toxicity of the free gold porphyrin nanoparticles.

\section{In vivo activity against neuroblastoma}

Subsequent study of in vivo antitumor kinetics was carried out. Tumor volume measurement was performed to investigate the in vivo efficacy of lipidic gold porphyrin nanoparticles. Tumor volume measurement was determined with the untreated and treated mice with increasing doses of lipidic gold porphyrin nanoparticles. Treatment of mice with $0.08 \mathrm{mg}$ or $0.1 \mathrm{mg}$ of lipidic gold porphyrin nanoparticles demonstrated a reduction of neuroblastoma growth compared with the untreated control (Figure 3a). Tumor size

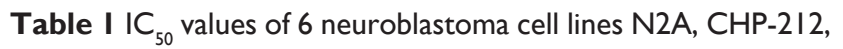
$\mathrm{Be}(2)-\mathrm{C}, \mathrm{SH}-\mathrm{SY} 5 \mathrm{Y}, \mathrm{LAN}-\mathrm{I}$, and LAN-2 treated with either free gold porphyrin nanoparticles (Free GP) or lipidic gold porphyrin nanoparticles (Lipidic GPNP)

\begin{tabular}{lll}
\hline Cell lines & Lipidic GPNP $\mathbf{~ m g} / \mathbf{m l}$ & Free GP $\mathbf{~ m g} / \mathbf{m l}$ \\
\hline N2A & 0.013 & 0.0032 \\
CHP-2I2 & 0.0075 & 0.0007 \\
Be(2)-C & 0.003 & 0.0006 \\
SH-SY5Y & 0.003 & 0.0012 \\
LAN-I & 0.0037 & 0.0076 \\
LAN-2 & 0.0034 & 0.0067 \\
\hline
\end{tabular}




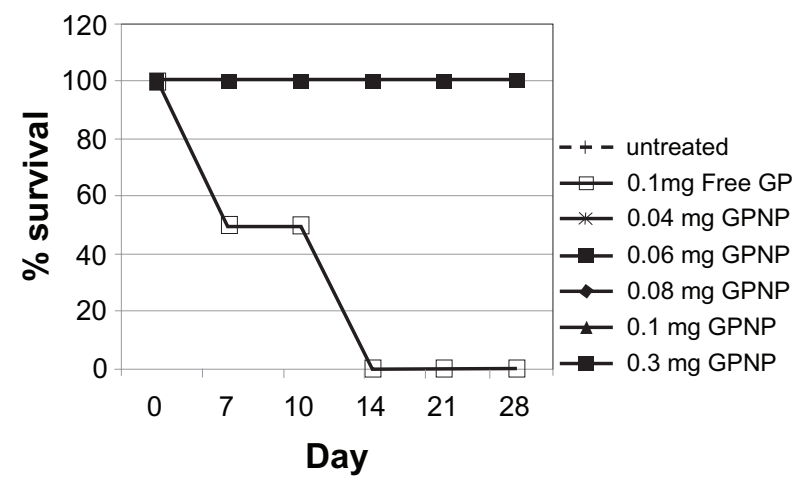

Figure 2 The effect of lipidic gold porphyrin nanoparticles on survival rate of normal A/J mice having received either free gold porphyrin nanoparticles or lipidic gold porphyrin nanoparticles. Data was represented in percentage of survival. GP represents free gold porphyrin nanoparticles while GPNP represents lipidic gold porphyrin nanoparticles. $(n=6)$.

following treatment with the $0.1 \mathrm{mg}$ or $0.08 \mathrm{mg}$ of lipidic gold porphyrin nanoparticles per week were approximately $14 \%$ (one-seventh) of the untreated tumor size (Figure 3a).

Furthermore, at the therapeutic doses of $0.08 \mathrm{mg}$ and $0.1 \mathrm{mg}$ lipidic gold porphyrin nanoparticles, tumor bearing mice survived up to 21 days at which time the animals were sacrificed. On the other hand, mice treated with $0.1 \mathrm{mg}$ of free gold porphyrin nanoparticles survived only 2 days (Figure $3 b$ ). This would suggest that the nanoparticle carrier was able to reduce the inherent toxicity of the free gold porphyrin nanoparticles. Nonetheless, at a higher dose of $0.1 \mathrm{mg}$, lipidic gold porphyrin nanoparticles still exhibited systemic toxicity. For the untreated mice, only $66 \%$ survived at day 21 , potentially due to the advanced and disseminated neuroblastoma (Figure 3b).

\section{Discussion}

Nanotechnology has received much attention in relation to drug delivery. Recent advances in the design of drug delivery system for cancer therapeutics have indicated platforms such as polymer micelles, lipidic carriers, and nanoparticles facilitate drug delivery with minimal toxicity. Traditional administration of free cancer therapeutics to neuroblastoma usually produces side effects. Long term and high dosage administration are required and this often causes accumulation in the kidneys and liver, which can cause irreversible damage to these organs and can therefore be life threatening.

To reduce toxicity, various strategies are required to facilitate the delivery of cancer therapeutics into the tumors. In the presence of the EPR effect, nanotechnology can be beneficial by coupling macromolecular carriers with small-size drugs. We found that the incorporation of lipidic carrier increases the neuroblastoma size 10 fold, with an average diameter
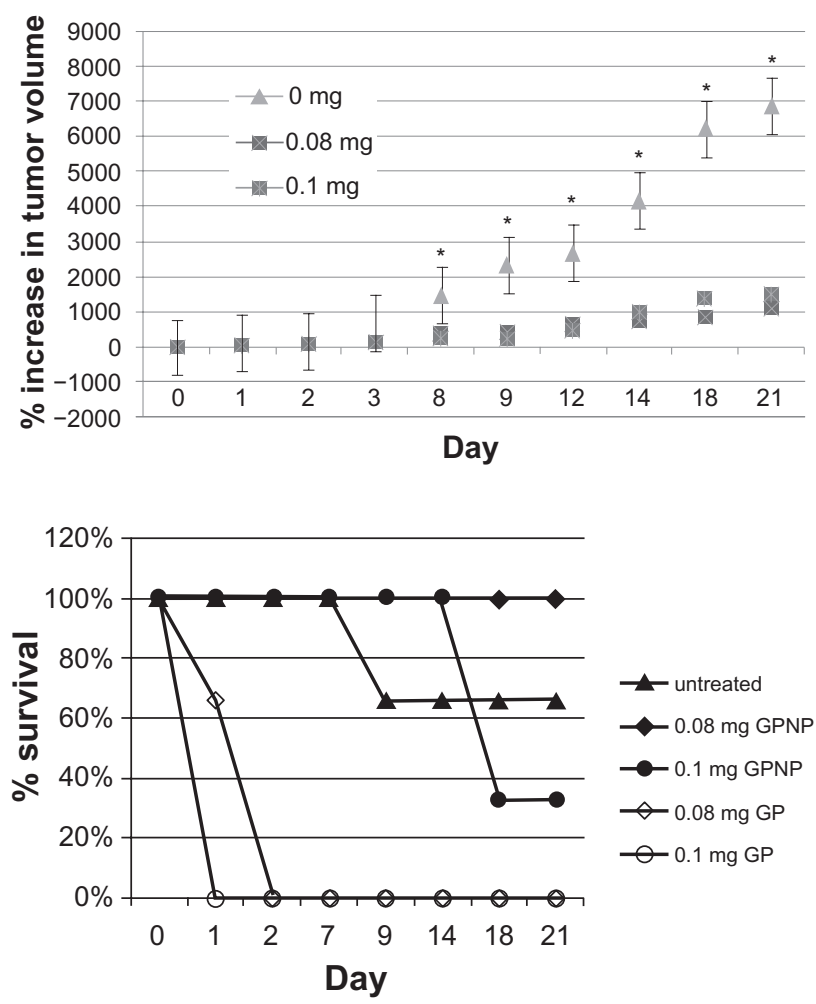

Figure 3 The therapeutic efficiency of lipidic gold porphyrin nanoparticles in neuroblastoma bearing mice. A) tumor volume measurements after tumorbearing mice received intraperitonal injection of $0.08 \mathrm{mg}$ or $0.1 \mathrm{mg}$ of lipidic gold porphyrin nanoparticles. $(n=5-6, * P<0.05)$. B) the effect of lipidic gold porphyrin nanoparticles on survival rate. $(n=6)$.

of approximately $80 \pm 30 \mathrm{~nm}$ (Figure 1). The increase in size can be favorable for drug accumulation in the tumor tissue based on EPR effect, as well as the slower lymphatic drainage. Through the size independent transfer from the peritoneal to bloodstream, nanoparticles can reach the tumor via the blood circulation..$^{20}$ Unlike small drugs which can be removed rapidly by the lymphatic system, macromolecules are too large to enter. In this case, the peritoneal cavity can serve as a drug depot for supporting the transfer to bloodstream. In fact, a significant increase in accumulation of large drugs was reported in the peritoneal cavity following the intraperitoneal route. ${ }^{21}$ In the normal vessel with a pore size of approximately $6 \mathrm{~nm}$ (17), the $80 \pm 30 \mathrm{~nm}$ nanoparticles cannot pass through the endothelial membrane. However, the pore of tumor vasculature could be as large as $400 \mathrm{~nm}$ and allow for the diffusion of the macromolecules into the tumor tissue. ${ }^{22}$ Due to the increased permeability of endothelial barrier in tumor vasculature, our delivery carrier is feasible for use in various administration routes via blood circulation, such as intravenously.

The importance of free gold porphyrin nanoparticles against neuroblastoma has been shown in previous reports 
of in vitro and preclinical studies. ${ }^{23}$ Consistent with previous findings, our data show that the free gold porphyrin nanoparticles are able to kill neuroblastoma cells. Our in vitro cytotoxicity examination demonstrated the effective killing of neuroblastoma cells using the carrier system. Interestingly, the $\mathrm{IC}_{50}$ value of lipidic gold porphyrin nanoparticles was much higher than free gold porphyrin nanoparticles. This may be due to the gold porphyrin being sequestered inside the nanoparticle carrier and needing time to be released. The different release kinetics of free gold porphyrin nanoparticles and lipidic gold porphyrin nanoparticles have been evidenced in a previous report involving liposome encapsulated drugs. ${ }^{24}$ Consistent with the in vitro cytotoxicity study, the intraperitoneal injection of lipidic gold porphyrin nanoparticles demonstrated an inhibition of the neuroblastoma growth (Figure 3). A possible explanation of the bioavailability inside the neuroblastoma tissue, despite the EPR effect, could be the chemical property of the nanoparticle carrier. The carrier consists of a fatty acid alcohol, cetyl alcohol. The incorporation of fatty acid alcohol into the lipid bilayer of the cell membrane occurs by lipid-lipid interaction. ${ }^{25}$ Results of the present study shed light on the mechanism of intracellular trafficking and internalization of lipidic carriers.

We observed different cytotoxicity in the six different neuroblastoma cell lines; this could be due to the different cell sensitivity to the gold porphryin. Since higher cytotoxicity of lipidic gold porphyrin nanoparticles was found in the LAN cell lines, it is possible that LAN is more sensitive to the lipidic gold porphryin nanoparticles, which would mean that it induced greater apoptosis. The different induction of apoptosis among the human neuroblastoma cell lines in response to drug was also evidenced in previous in vitro study. ${ }^{26}$

The potential benefits in clinical application can be revealed by ruling out the toxicity associated in a biological system. The $\mathrm{A} / \mathrm{J}$ mouse strain is a common model for neuroblastoma study. ${ }^{27,28}$ However, the $\mathrm{A} / \mathrm{J}$ mouse strain appears to show higher susceptibility to gold porphyrin compared with other strains such as $\mathrm{BALB} / \mathrm{c}^{29}$ or $\mathrm{C} 57 \mathrm{Bl} / 6$ (unpublished data). This was seen in our in vivo study that all the A/J mice died 2 days after administration of free gold porphyrin nanoparticles. By incorporation of lipidic carrier, A/J mice survived even after three repeated dosage administrations of gold porphyrin (Figure 2). As chemotherapeutics or radiotherapeutics often require repeated administrations, this delivery carrier can be beneficial in the cancer treatment by reducing the side effects. Further in vivo study is thus warranted.

Despite the reduced toxicity by the lipidic nanoparticle carrier, improvement in the design is necessary to increase the potency in clinical applications. The survival study of the neuroblastoma bearing mice showed that $100 \%$ of mice survived a dose of $0.08 \mathrm{mg}$ of lipidic gold porphyrin nanoparticles, but the survival rate dropped to $33 \%$ for a dose of $0.1 \mathrm{mg}$ gold porphyrin nanoparticles by the time 21 days had elapsed. Thus toxicity could still be present if high therapeutic doses of the drug are necessary for treatment. One strategy is the attachment of the specific tumor targeting ligand to the surface of current lipidic nanoparticle carrier. This active tumor targeting strategy has been shown to be useful in delivery of cancer therapeutics by further facilitating the accumulation and cellular uptake inside tumors. ${ }^{30}$

In conclusion, gold porphyrin incorporated with a nanoparticle lipidic carrier demonstrated an antitumor activity with reduced toxicity. We therefore conclude that this is a useful strategy in developing a safe delivery system for cancer chemotherapeutics.

\section{Acknowledgments}

The work was supported by HKU Small Project Funding \#200907176012 and the Area of Excellence Scheme (AoE/P-10/01) established under the University Grants Committee (C-M Che). The authors would like to thank the Electron Microscopy Unit at Department of Pathology at HKU for providing assistance with electron microscopy.

\section{Disclosure}

The authors report no conflicts of interest in this work.

\section{References}

1. Laverdiere C, Liu, Q, Yasui, Y, et al. Long-term outcomes in survivors of neuroblastoma: a report from the Childhood Cancer Survivor Study. J Natl Cancer Inst. 2009;101:1131-1140.

2. Matthay KK, Villablanca, JG, Seeger, RC, et al. Treatment of high-risk neuroblastoma with intensive chemotherapy, radiotherapy, autologous bone marrow transplantation, and 13-cis-retinoic acid. Children's Cancer Group. N Engl J Med. 1999;341:1165-1173.

3. Rybak LP, Mukherjea D, Jajoo S, et al. Cisplatin ototoxicity and protection: clinical and experimental studies. Tohoku J Exp Med. 2009;219:177-186.

4. Kim E, Moore J, Huang J, et al. All angiogenesis is not the same: Distinct patterns of response to antiangiogenic therapy in experimental neuroblastoma and Wilms tumor. J Pediatr Surg. 2001;36:287-290.

5. Matsumura Y, Maeda H. A new concept for macromolecular therapeutics in cancer-chemotherapy - mechanism of tumoritropic accumulation of proteins and the antitumor agent SMANCS. Canc Res. 1986;46:6387-6392.

6. Maeda H, Matsumura Y. Tumoritropic and lymphotropic principles of macromolecular drugs. Crit Rev Ther Drug Carr Sys. 1989;6: $193-210$

7. Jun YJ, Kim JI, Jun MJ, Sohn YS. Selective tumor targeting by enhanced permeability and retention effect. Synthesis and antitumor activity of polyphosphazene-platinum (II) conjugates. J Inorg Biochem. 2005;99:1593-1601. 
8. Maeda H. The enhanced permeability and retention (EPR) effect in tumor vasculature: the key role of tumor-selective macromolecular drug targeting. Adv Enz Reg. 2001;41:189-207.

9. Tanaka T, Shiramoto S, Miyashita M, et al. Tumor targeting based on the effect of enhanced permeability and retention (EPR) and the mechanism of receptor-mediated endocytosis (RME). Int J Pharm. 2004;277:39-61.

10. Che CM, Sun R, Yu WY, et al. Gold(III) porphyrins as a new class of anticancer drugs: cytotoxicity, DNA binding and induction of apoptosis in human cervix epitheloid cancer cells. Chem Commun. 2003;14:1718-1719.

11. Sun RWY, Che CM, The anti-cancer properties of gold(III) compounds with dianionic porphyrin and tetradentate ligands. Chem Rev. 2009;253:1682-1691.

12. Shaw IC. Gold-based therapeutic agents. Chem Rev. 1999;99: 2589-2600.

13. To YF, Sun RWY, Chen Y, et al. Gold(III) porphyrin complex is more potent than cisplatin in inhibiting growth of nasopharyngeal carcinoma in vitro and in vivo. Int J Cancer. 2009;124:1971-1979.

14. Bindoli A, Rigobello MP, Scutari G, et al. Thioredoxin reductase: a target for gold compounds acting as potential anticancer drugs. Chem Rev. 2009;253:1692-1707.

15. Tu S, Sun RWY, Lin MCM, et al. Gold(III) porphyrin complexes induce apoptosis and cell cycle arrest and inhibit tumor growth in colon cancer. Cancer. 2009;115:4459-4469.

16. Wang Y, He QY, Sun RWY, et al. Gold(III) porphyrin 1a induced apoptosis by mitochondrial death pathways related to reactive oxygen species. Cancer Res. 2005;65:11553-11564.

17. Koziara JM, Oh JJ, Akers WS, et al. Blood compatibility of cetyl alcohol/ polysorbate-based nanoparticles. Pharm Res. 2005;22:1821-1828.

18. Akiyama Y, Mori T, Katayama Y, Niidome T. The effects of PEG grafting level and injection dose on gold nanorod biodistribution in the tumor-bearing mice. $J$ Cont Rel. 2009;139:81-84.

19. Hsu CH, Cui Z, Mumper RJ, Jay M. Preparation and characterization of novel coenzyme Q10 nanoparticles engineered from microemulsion precursors. AAPS Pharm Sci Tech. 2003;4:E32.

20. Seymour LW, Duncan R, Strohalm J, et al. Effect of molecular-weight (mbarw) of N-(2 hydroxypropyl)methacrylamide copolymers on body distribution and rate of excretion after subcutaneous, intraperitoneal, and intravenous administration to rats. $J$ Biomed Mat Res. 1987;21:1341-1358.
21. Nagy JA, Herzberg KT, Masse EM, et al. Exchange of macromolecules between plasma and peritoneal cavity in ascites tumor-bearing, normal, and serotonin-injected mice. Cancer Res. 1989;49:5448-5458.

22. Yuan F, Dellian M, Fukumura D, et al. Vascular permeability in a human tumor xenograft: molecular size dependence and cutoff size. Cancer Res. 1995;55:3752-3756.

23. Li W, Xie Y, Sun RWY, et al. Inhibition of Akt sensitises neuroblastoma cells to gold(III) porphyrin 1a, a novel antitumour drug induced apoptosis and growth inhibition. Br J Cancer. 2009;101:342-349.

24. Wu J, Lu Y, Lee A, et al. Reversal of multidrug resistance by transferrinconjugated liposomes co-encapsulating doxorubicin and verapamil. J Pharm Pharm Sci. 2007;10:350-357.

25. Grunze M, Haest CW, Deuticke B. Lateral segregation of membrane lipids and formation of stable rod-shaped membrane projections in erythrocytes treated with long-chain alcohols. Biochem Biophys Acta. 1982;693:237-245

26. Schmidt ML, Kuzmanoff KL, Ling-Indeck L, et al. Betulinic acid induces apoptosis in human neuroblastoma cell lines. Eur J Cancer. 1997;33:2007-2010.

27. Auf G, Chen L, Fornès P, et al. CpG-oligodeoxynucleotide rejection of a neuroblastoma in $\mathrm{A} / \mathrm{J}$ mice does not induce a paraneoplastic disease. Neurosci Let. 2002;327:189-192.

28. Katsanis E, Blazar BR, Bausero MA, et al. Retroperitoneal inoculation of murine neuroblastoma results in a reliable model for evaluation of the antitumor immune-response. J Ped Surg. 1994;29:538-542.

29. Li W, Xie Y, Sun RWY, et al. Inhibition of Akt sensitises neuroblastoma cells to gold(III) porphyrin 1a, a novel antitumour drug induced apoptosis and growth inhibition. Brit J Canc. 2009;101:342-349.

30. Bartlett DW, Su H, Hildebrandt IJ, et al. Impact of tumor-specific targeting on the biodistribution and efficacy of siRNA nanoparticles measured by multimodality in vivo imaging. Proc Nat Acad Sci USA. 2007;104:15549-15554.
Nanotechnology, Science and Applications

\section{Publish your work in this journal}

Nanotechnology, Science and Applications is an international, peerreviewed, open access journal that focuses on the science of nanotechnology in a wide range of industrial and academic applications. It is characterized by the rapid reporting across all sectors, including engineering, optics, bio-medicine, cosmetics, textiles, resource sustainability

\section{Dovepress}

and science. Applied research into nano-materials, particles, nanostructures and fabrication, diagnostics and analytics, drug delivery and toxicology constitute the primary direction of the journal. The manuscript management system is completely online and includes a very quick and fair peer-review system, which is all easy to use. 